\title{
Spin-1/2 XX chains in a transverse field with regular nonuniformity or correlated Lorentzian disorder
}

\author{
O.Derzhko, T.Krokhmalskii, O.Zaburannyi \\ Institute for Condensed Matter Physics \\ of the National Academy of Sciences of Ukraine, \\ 1 Svientsitskii Str., 290011 Lviv, Ukraine
}

Received June 11, 1998, in final form June 24, 1998

\begin{abstract}
Using continued fractions we examined the density of states and thermodynamic properties of a few periodic nonuniform spin- $1 / 2 X X$ chains in a transverse field. We considered the transverse spin dynamics in spin-1/2 $\mathrm{XX}$ chain with correlated Lorentzian disorder.
\end{abstract}

Key words: periodic nonuniform spin-1/2 XX chain, correlated Lorentzian disorder, density of states, magnetization, specific heat, dynamic structure factor

PACS: $75.10 .-b$

Since the pioneering paper by Lieb, Schultz and Mattis [1] statistical mechanics calculations for spin- $\frac{1}{2} X Y$ chains have been the subject of long-lasting interest both from fundamental and applied viewpoints. Our communication deals with some new results for thermodynamics and dynamics of isotropic transverse $X Y$ chains with regular nonuniformity or correlated Lorentzian disorder. The former model generalizes the $X X$ chain with the alternating coupling constant that was investigated by some authors as a toy model to consider spin-Peierls phase transition [2-4]. The latter model was studied in [5]. However the treatment in that paper was restricted to thermodynamics in contrast to the present study dealing with dynamics of transverse spin correlations. The basic tools to study these models are the Jordan-Wigner method, the continued fractions, and the numerical approach for examining the spin correlation dynamics developed recently [6].

Hereinafter we investigate a nonuniform $X X$ chain in a magnetic field along the $z$-axis consisting of $N$ spins $\frac{1}{2}$ wherein Hamiltonian is defined by

$$
H=\sum_{n} \Omega_{n} s_{n}^{z}+\sum_{n} J_{n}\left(s_{n}^{x} s_{n+1}^{x}+s_{n}^{y} s_{n+1}^{y}\right)
$$




$$
=\sum_{n} \Omega_{n}\left(s_{n}^{+} s_{n}^{-}-\frac{1}{2}\right)+\sum_{n} I_{n}\left(s_{n}^{+} s_{n+1}^{-}+s_{n}^{-} s_{n+1}^{+}\right) .
$$

By the Jordan-Wigner transformation the Hamiltonian (1) can be represented as the Hamiltonian of non-interacting spinless fermions

$$
H=\sum_{n} \Omega_{n}\left(c_{n}^{+} c_{n}-\frac{1}{2}\right)+\sum_{n} I_{n}\left(c_{n}^{+} c_{n+1}-c_{n} c_{n+1}^{+}\right) .
$$

To examine the thermodynamics of the model one must find the density of magnon states $\rho(E)$ that is related to the temperature double-time fermion Green functions $G_{n m}^{\mp} \equiv G_{n m}^{\mp}(E \pm \mathrm{i} \epsilon)$ according to the formula

$$
\rho(E)=\mp \frac{1}{\pi N} \sum_{n=1}^{N} \operatorname{Im} G_{n n}^{\mp} .
$$

Using the equation of motion for $G_{n m}^{\mp}$ it is a simple matter to show that

$$
\begin{aligned}
G_{n n}^{\mp} & =\frac{1}{E \pm \mathrm{i} \epsilon-\Omega_{n}-\Delta_{n}^{-}-\Delta_{n}^{+}}, \\
\Delta_{n}^{-} & =\frac{I_{n-1}^{2}}{E \pm \mathrm{i} \epsilon-\Omega_{n-1}-\frac{I_{n-2}^{2}}{E \pm \mathrm{i} \epsilon-\Omega_{n-2}-} \cdot} \\
\Delta_{n}^{+} & =\frac{I_{n}^{2}}{E \pm \mathrm{i} \epsilon-\Omega_{n+1}-\frac{I_{n+1}^{2}}{E \pm \mathrm{i} \epsilon-\Omega_{n+2}-} \cdot} .
\end{aligned}
$$

The continued fraction representation for the diagonal Green functions (4) is extremely useful for examining thermodynamics of regularly nonuniform chains when periodic continued fractions emerge.

Consider for example a regular alternating chain $\Omega_{1} I_{1} \Omega_{2} I_{2} \Omega_{1} I_{1} \Omega_{2} I_{2} \ldots$ when periodic continued fractions with period 2 emerge. As a result

$$
\rho(E)= \begin{cases}0, & E<b_{4}, \quad b_{3}<E<b_{2}, \quad b_{1}<E, \\ \frac{1}{2 \pi} \frac{\left|2 E-\Omega_{1}-\Omega_{2}\right|}{\sqrt{\mathcal{B}(E)}}, & b_{4}<E<b_{3}, \quad b_{2}<E<b_{1} .\end{cases}
$$

Here

$$
\begin{aligned}
\mathcal{B}(E) & =4 I_{1}^{2} I_{2}^{2}-\left[\left(E-\Omega_{1}\right)\left(E-\Omega_{2}\right)-I_{1}^{2}-I_{2}^{2}\right]^{2} \\
& =-\left(E-b_{1}\right)\left(E-b_{2}\right)\left(E-b_{3}\right)\left(E-b_{4}\right) \\
\left\{b_{i}\right\} & =\left\{\frac{1}{2}\left[\Omega_{1}+\Omega_{2} \pm \sqrt{\left(\Omega_{1}-\Omega_{2}\right)^{2}+4\left(I_{1} \pm I_{2}\right)^{2}}\right]\right\}
\end{aligned}
$$

and in (5), (6) it is implied that $b_{1} \geqslant b_{2} \geqslant b_{3} \geqslant b_{4}$. In principle the calculation of $\rho(E)$ can be done for an arbitrary periodic chain, however, the calculations in 

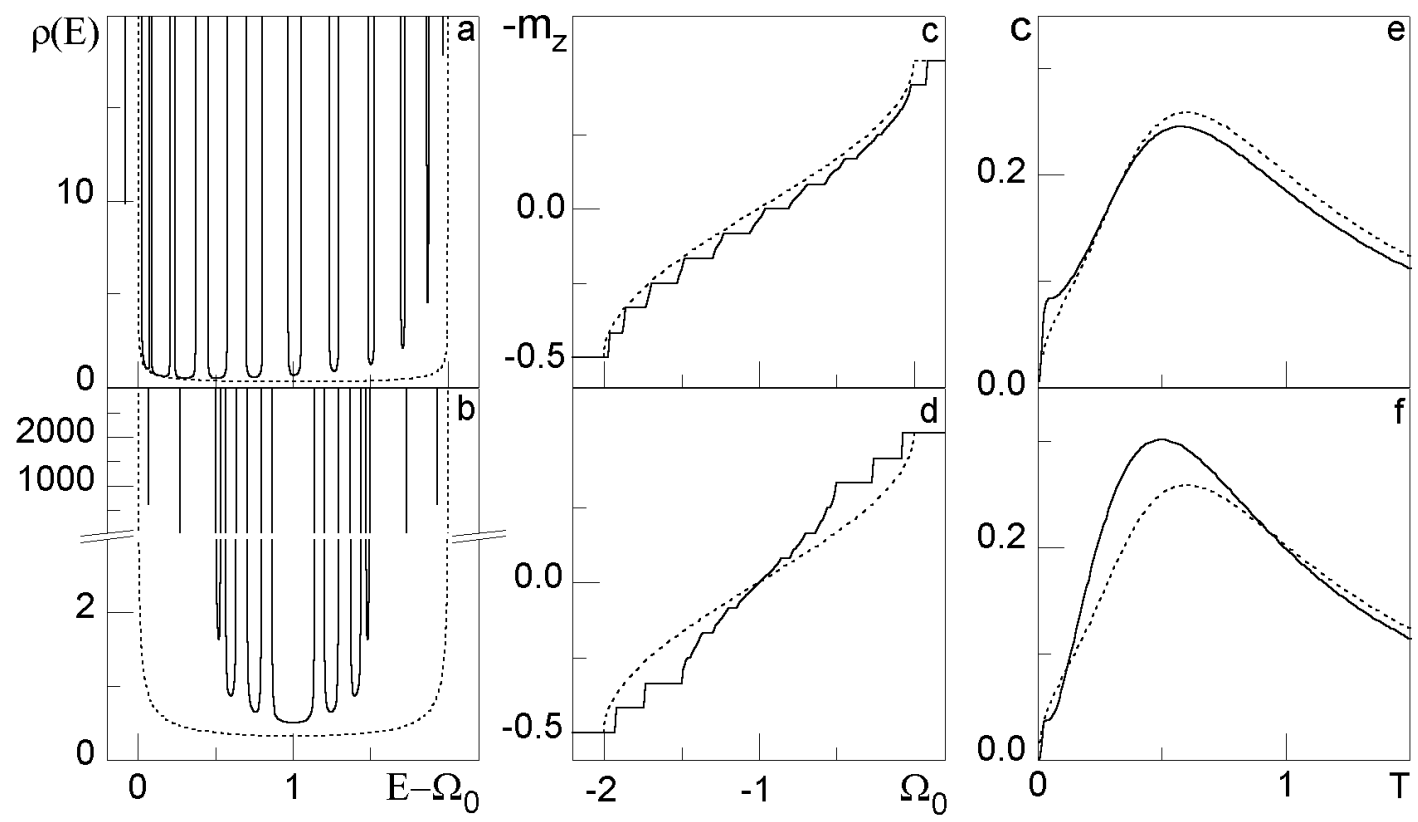

Figure 1. Density of states $(a, b)$, transverse magnetization versus transverse field at zero temperature $\beta=\infty(\mathrm{c}, \mathrm{d})$ and temperature dependence of specific heat (e, f) for nonuniform chain $\Omega_{1} I_{1} \ldots \Omega_{12} I_{12} \Omega_{1} I_{1} \ldots \Omega_{12} I_{12} \ldots \Omega_{1}=\Omega_{12}=$ $0.5+\Omega_{0}, \Omega_{2}=\ldots=\Omega_{11}=1+\Omega_{0}, J_{1}=\ldots=J_{11}=1, J_{12}=0.5(\mathrm{a}, \mathrm{c}, \mathrm{e}) ;$ $\Omega_{1}=\ldots=\Omega_{12}=1+\Omega_{0}, J_{1}=\ldots=J_{6}=1, J_{7}=\ldots=J_{12}=0.5(\mathrm{~b}, \mathrm{~d}, \mathrm{f}) ;$ dotted curves correspond to uniform case.

the case of large periods become cumbersome. In figures 1a, $1 \mathrm{~b}$ we plotted $\rho(E)$ for two chains with the period 12 . The main result of introducing the regular nonuniformity is a spliting of the magnon band into subbands (a number of subbands is equal to or less than the period of nonuniformity; compare figure 1a and figure $1 \mathrm{~b}$ ) that has important consequences in the thermodynamic properties of spin model. For instance the low-temperature dependence of transverse magnetization $m_{z}=-\frac{1}{2} \int_{-\infty}^{\infty} \mathrm{d} E \rho(E) \tanh \frac{\beta E}{2}$ on transverse field is made up of sharply increasing parts and horizontal parts, their number being determined by the period of nonuniformity (figures 1c, 1d). In figures 1e, 1f we plotted the temperature dependence of specific heat $c=\int_{-\infty}^{\infty} \mathrm{d} E \rho(E)\left(\frac{\beta E}{2}\right)^{2} / \cosh ^{2} \frac{\beta E}{2}$ that due to the introduced periodic nonuniformity exhibits a two-peak structure, i.e. it has low-temperature and high-temperature peaks.

Let us consider spin model (1) assuming that the exchange couplings $J_{n}$ are independent Lorentzian variables with distribution

$$
p\left(J_{n}\right)=\frac{1}{\pi} \frac{\Gamma}{\left(J_{n}-J_{0}\right)^{2}+\Gamma^{2}}
$$

( $J_{0}$ is the mean value of exchange coupling and $\Gamma$ is the width of distribution that controls the strength of disorder) and the transverse fields are determined by the 

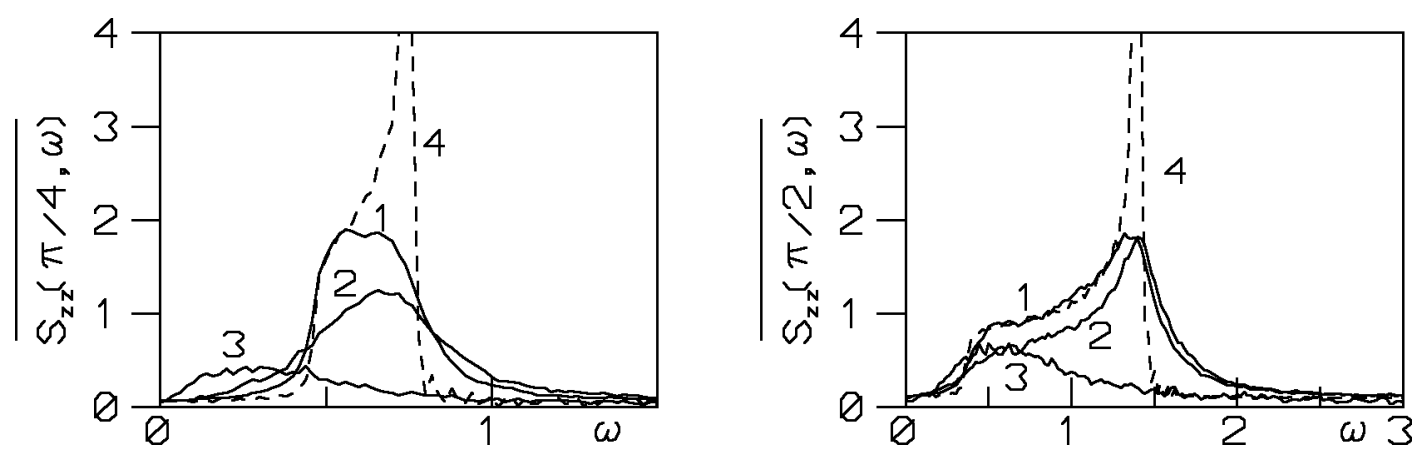

Figure 2. Frequency dependence of $\overline{S_{z z}(\kappa, \omega)}(10)$ at $\kappa=\frac{\pi}{4}$ and $\kappa=\frac{\pi}{2} .1-$ correlated disorder with $a=-1.01 ; 2$ - correlated disorder with $a=1.01 ; 3$ independent exchange couplings and transverse fields; the latter have distribution (9) with $|a| \Gamma=0.101 ; 4$ - non-random case $\Gamma=0$ (dashed curves).

neighbouring exchange couplings according to the formula

$$
\Omega_{n}-\Omega_{0}=\frac{a}{2}\left(J_{n-1}+J_{n}-2 J_{0}\right) .
$$

It can readily be checked that the distribution for the random variable $\Omega_{n}$ reads

$$
p\left(\Omega_{n}\right)=\frac{1}{\pi} \frac{|a| \Gamma}{\left(\Omega_{n}-\Omega_{0}\right)^{2}+(|a| \Gamma)^{2}} .
$$

We shall be interested in calculation of the random-averaged dynamic structure factor

$$
\left.\overline{S_{z z}(\kappa, \omega)}=\sum_{n} \mathrm{e}^{\mathrm{i} \kappa n} \int_{-\infty}^{\infty} \mathrm{d} t \mathrm{e}^{-\epsilon|t|} \mathrm{e}^{\mathrm{i} \omega t} \overline{\left\langle\overline{\left.s_{j}^{z}(t) s_{j+n}^{z}\right\rangle}\right.}-\overline{\left\langle s_{j}^{z}\right\rangle\left\langle s_{j+n}^{z}\right\rangle}\right]
$$

using for this purpose numerical approach. As shown in [6], to achieve this goal it is necessary to solve $N \times N$ eigenvalue and eigenvector problem for the matrix $A_{n m}=\Omega_{n} \delta_{n m}+\frac{J_{n}}{2} \delta_{m, n+1}+\frac{J_{n-1}}{2} \delta_{m, n-1}$, i.e.

$$
\sum_{j=1}^{N} g_{k j} A_{j m}=\Lambda_{k} g_{k m}, \quad \sum_{j=1}^{N} g_{k j} g_{q j}=\delta_{k q}, \quad \sum_{k=1}^{N} g_{k j} g_{k m}=\delta_{j m} .
$$

In our numerical calculations we considered chains of $N=300$ spins with $J_{0}=-1$, $\Omega_{0}=0.5$ and $\Gamma=0.1$ at low temperature $\beta=1000$. We computed correlation functions $\left\langle s_{150}^{z}(t) s_{150+n}^{z}\right\rangle-\left\langle s_{150}^{z}\right\rangle\left\langle s_{150+n}^{z}\right\rangle$ with $n=0, \pm 1, \ldots, \pm 100$ for the times up to $t=200$, put $\epsilon=0.01$ and averaged the $z z$ dynamic structure factor (10) over 3000 random realizations to obtain the results presented in figure 2 . We carefully analyzed the accuracy of our calculations studying finite-size effects, the effects of finite number of terms in the sum in (10) and of finite time cut-off in the integral in (10), and the effects of finite number of random realizations.

Let us comment the results we have obtained for $\overline{S_{z z}(\kappa, \omega)}$. Figure 2 nicely shows the difference in frequency shapes of the dynamic structure factor for correlated 


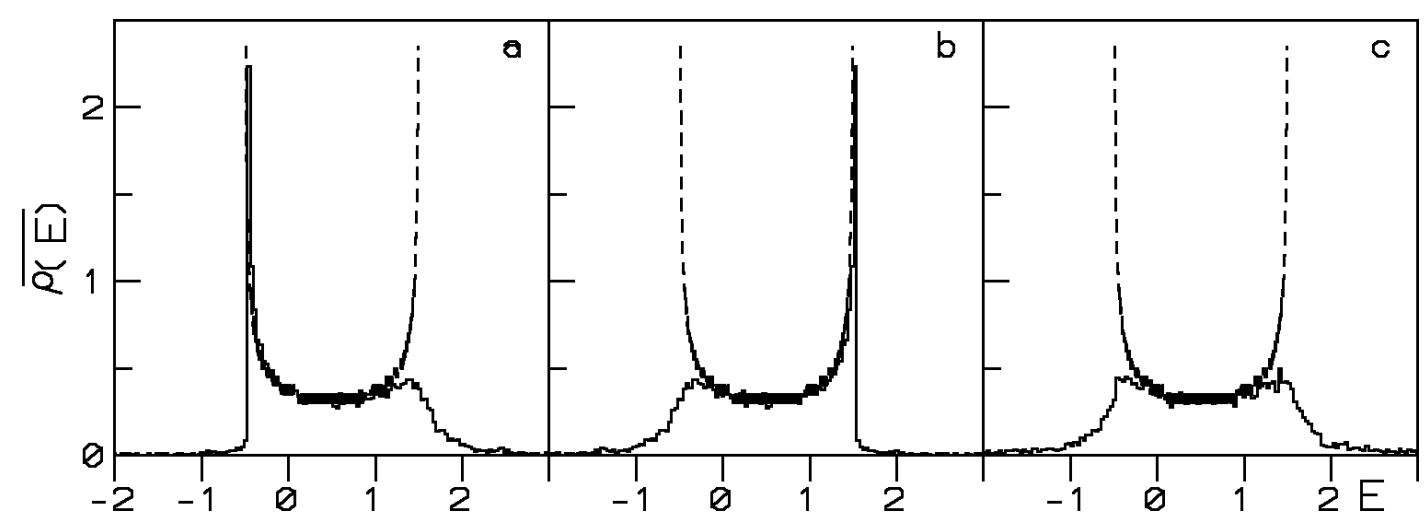

Figure 3. Density of states for model (1), (7). a) - correlated disorder with $a=-1.01$; b) - correlated disorder with $a=1.01 ; \mathrm{c}$ ) - independent exchange couplings and transverse fields with $|a| \Gamma=0.101$. The density of states for nonrandom case is depicted by dashed curves.

disorder (7), (8) with different signs of $a$ and for the case of independent random exchange couplings and transverse fields with distributions (7) and (9), respectively. The transverse dynamic structure factor is determined by two magnon excitations and these spectacular changes in the frequency dependence are caused by the changes in the density of magnon states depicted in figure 3 .

To summarize, we applied continued fractions to study rigorously the thermodynamic properties of periodic nonuniform spin- $\frac{1}{2} X X$ chain in a transverse field and extended a previous analysis of the spin- $\frac{1}{2} X X$ chain with correlated Lorentzian disorder examining numerically the dynamics of transverse spin correlations. The theoretical results observed in our study should prove valuable in understanding the experimental data for $X X$ chain materials the synthesis of which is becoming a reality [7].

A study of periodic nonuniform spin- $\frac{1}{2} X X$ chains was inspired by the papers of J. Freericks and R. Lemański presented at the 22nd Seminar of the Middle European Cooperation in Statistical Physics (Szklarska Porȩba, 1997). The authors thank R. Lemański for useful correspondence. O. D. is indebted to Mr. Joseph Kocowsky for continuous financial support.

\section{References}

1. Lieb E., Schultz T., Mattis D. Two soluble models of an antiferromagnetic chain. // Ann. Phys. (N.Y.), 1961, vol. 16, p. 407-466.

2. Pincus P. Instability of the uniform antiferromagnetic chain. // Solid State Commun., 1971, vol. 9, p. 1971-1973.

3. Lima R.A.T., Tsallis C. Magnetic field influence on the spin-Peierls instability in the quasi-one-dimensional magnetostrictive $X Y$ model: Thermodynamical properties. // Phys. Rev. B, 1983, vol. 27, No. 11, p. 6896-6915.

4. Okamoto K. Alternating $s=\frac{1}{2} X Y$ chain in the Lorentzian random field. // J. Phys. Soc. Jpn., 1990, vol. 59, No. 12, p. 4286-4296. 
5. Derzhko O., Richter J. Solvable model of random spin- $\frac{1}{2} X Y$ chain. // Phys. Rev. B, 1997, vol. 55, No. 21, p. 14298-14310.

6. Derzhko O., Krokhmalskii T. Numerical approach for a study of the spin- $\frac{1}{2} X Y$ chains dynamic properties. // Phys. Status Solidi B, 1998, vol. 208, No. 1, p. 221-248.

7. Collins M.F., Petrenko O.A. Triangular antiferromagnets. Preprint, condmat/9706153, 1997.

\title{
Спін-1/2 XX ланцюжки в поперечному полі з регулярною неоднорідністю або із скорельованим лоренцовим безладом
}

\author{
О.Держко, Т.Крохмальський, О.Забуранний \\ Інститут фізики конденсованих систем НАН України, \\ 290011 Львів, вул. Свєнціцького, 1 \\ Отримано 11 червня 1998 р., в остаточному вигляді - \\ 24 червня 1998 р.
}

Використовуючи неперервні дроби, ми дослідили щільність станів і термодинамічні властивості кількох періодично неоднорідних спін1/2 XX ланцюжків у поперечному полі. Ми розглянули поперечну спінову динаміку в спін-1/2 XX ланцюжку із скорельованим лоренцовим безладом.

Ключові слова: періодично неоднорідний спін-1/2 XX ланцюжок, скорельований лоренців безлад, щільність станів, намагніченість, теплоємність, динамічний структурний фактор

PACS: $75.10 .-b$ 